\title{
Accuracy assessment of solving pseudo compressible euler equations for steady subsonic flow on finite unstructured volumes
}

\author{
S. R. Sabbagh Yazdi* A. Hadian ${ }^{\dagger}$
}

(Received 18 October 2004, revised 7 November 2005)

\begin{abstract}
The inviscid incompressible Euler equations are applied to a wide range of engineering applications. For steady state problems, the equation of continuity can be simultaneously solved with the equations of motion in a coupled manner using the pseudo compressibility method. This method helps couple the pressure and the velocity fields during the explicit computation procedure, and therefore speeds the convergence of the method. The discrete form of the two-dimensional flow equations are formulated using the Cell Vertex Finite Volume Method for triangular unstructured meshes. Using triangular unstructured
\end{abstract}

*Assistant Prof. of Civil Engineering Department, KN Toosi University of Technology, Tehran, IrAN, mailto:SYazdi@kntu.ac.ir

${ }^{\dagger}$ MSc Graduate Student of Civil Engineering Department, KN Toosi University of Technology, Tehran, Iran.

See http://anziamj.austms.org.au/V46/CTAC2004/Yazd for this article, (c) Austral. Mathematical Soc. 2005. Published November 16, 2005. ISSN 1446-8735 
meshes provides great flexibility for modelling flow in geometrically complex domains. Some numerical methods adopted for unstructured meshes stabilize and accelerate the explicit solution procedure without degrading the accuracy. In order to verify the accuracy of the numerical scheme, computed results are compared with the exact solution for two potential flow problems.

\section{Contents}

1 Introduction

C1223

2 Pseudo compressibility method

C1225

3 Numerical method

C1227

4 Boundary conditions

C1229

5 Domain discretization

C1230

6 Verification of accuracy

C1230

7 Discussion

C1235

References

$\mathrm{C} 1237$

\section{Introduction}

The assumption of incompressibility is valid for common civil and environmental engineering problems. For the most civil engineering flow problems, the boundary layer is confined to thin regions close to the solid surfaces. Since these regions are negligible compared to the main domain of interest, 
the effect of viscous stresses can be omitted in the equations of the motion. The resulting set of equations, which is known as the incompressible form of the Euler equations, provides considerable simplicity in the absence of second order spatial derivative terms. This simplification of the governing equations makes the solution easier, and consequently reduces the computational effort.

For incompressible flow conditions, the time derivative of the density vanishes from the continuity equation. If the boundary layer thickness is negligible in the flow domain, the inviscid form of the equations of motion can be used. This set of equations, consisting of the time-independent continuity equation and the time-dependent equations of motion, mathematically represent the behavior of fluid flow. For steady state problems, adding a pseudo time derivative of pressure to the continuity equation removes the troublesome problem of coupling pressure-independent equation of continuity to the pressure-dependent equations motion. This method has been widely applied, mostly with the use of explicit schemes. The computational procedure is to choose the pressure field such that continuity is satisfied at each time-step. This procedure normally requires a relaxation scheme iterating on pressure until the divergence free condition is reasonably realized. The method of pseudo compressibility was initially proposed by Chorin [2] to achieve an efficient computation.

In the present work, the Cell Vertex Finite Volume Method is used to derive the discrete formulas of the governing equations on triangular unstructured meshes. The problem of growing numerical errors, which usually disturb the explicit solution, are overcome by adding artificial dissipation terms suitable for unstructured meshes. These extra terms are used to damp out the unwanted errors and stabilize the numerical solution procedure while preserving the accuracy of the solution. In order to increase the computational efficiency, numerical methods such as Runge-Kutta multi-stage time stepping, residual smoothing and the edge-base algorithm are employed. The accuracy of the described algorithm for the solution of the inviscid incompressible flow equations is assessed by simulation of some simple test cases for 
which the analytical solution of the velocity and pressure field are obtained. The accuracy is demonstrated by comparing exact and computed velocity and pressure fields.

\section{Pseudo compressibility method}

For flow with a high Reynolds number, the boundary layer is thin and limited to a thin layer close to solid walls. In such cases, the effects of viscosity may be ignored in the major part of the flow field. The assumption of inviscid behavior of the fluid flow is acceptable for the regions outside the boundary layer. For low-speed flow problems (Mach number $<0.3$ ) even a fluid such as air is effectively incompressible and hence its density is constant for isothermal conditions.

Due to negligible variation in the density, there is no time derivative term in the continuity equation. This matter presents some numerical difficulties for the coupled solution of the continuity equation (zero velocity divergence) together with some time dependent equations (the equations with pressure and velocity components as the dependent variables). For the steady state incompressible problems, the pseudo compressibility method helps to overcome this numerical solution problem [2]. In this method, a time derivative of the pressure, which is derived from an equation similar to the equation of state of compressible gases, is added to the continuity equation. This transient term, which relates the pressure field to the velocity field, vanishes when the solution converges to the steady state condition.

The conservative vector form of the governing equations in Cartesian coordinates is

$$
\frac{\partial W}{\partial t}+\frac{\partial F}{\partial x}+\frac{\partial G}{\partial y}=0
$$

where $W=\left\{p /\left(\rho_{0} \beta^{2}\right), u, v\right\}^{T}$ represents the conserved variables, and $F=$ $\left\{u, u^{2}+p / \rho_{0}, u v\right\}^{T}$ and $G=\left\{v, v u, v^{2}+p / \rho_{0}\right\}^{T}$ are vectors of convective 
fluxes of $W$ in $x$ and $y$ directions respectively. The components of velocity $u$ and $v$ and pressure $p$ are three dependent variables, and $\rho_{0}$ is the constant density. The parameter $\beta$ is introduced, using the analogy to the speed of sound in the equation of state of a compressible fluid, by application of the pseudo compressibility method [2].

In above equations the first row presents the incompressible continuity equation modified according to the pseudo compressibility method. The second and third rows correspond to the equations of motion in the $x$ and $y$ directions respectively.

The system of equations governing the motion of an incompressible flow is of the elliptic type. In an elliptic formulation, pressure waves propagate with infinite speed. Using the pseudo compressibility method, waves of finite speed are introduced into the incompressible flow field as a mechanism to distribute the pressure. Therefore, the system of modified equations given by the modified continuity equation and equations of motion is of hyperbolic type. Chang and Kwak [1] present the theory for the method of pseudo compressibility method.

The speed of propagation of these pseudo waves depends on the magnitude of the pseudo compressibility parameter. Ideally, the value of the pseudo compressibility parameter is chosen so that the speed of the introduced waves approaches that of the incompressible flow. However, this introduces a problem of contaminating the accuracy of the numerical algorithm, as well as affecting the stability property. On the other hand, if the pseudo compressibility parameter is chosen such that these waves travel too slowly, then the variation of the pressure field accompanying these waves is very slow. Therefore, a method of controlling the speed of pressure waves is a key to the success of this approach.

In the present formulation, the magnitude of the wave speed depends on the parameter $\beta$. Therefore, the success of the present method depends on the value of $\beta$ that must be used for fast convergence to steady-state solu- 
tions, and whether the incompressibility is really achieved within a desired accuracy by the use of the above equations. Some algorithms used a constant value of the pseudo compressibility parameter, and some workers developed sophisticated algorithms for solving mixed incompressible and compressible problems [9]. However, the value of the parameter may be considered as a function of local velocity using the following formula proposed by Dreyer [3]:

$$
\beta^{2}=\max \left(\beta_{\min }^{2}, \alpha U^{2}\right) .
$$

In order to prevent numerical difficulties in the region of very small velocities (that is, in the vicinity of stagnation points), the parameter $\beta_{\min }^{2}$ is considered in the range of 0.1 to 0.3 , and optimum $\alpha$ is suggested between 1 and 5 [9]. In the present work, the parameters $\beta_{\min }^{2}$ and $\alpha$ are 0.25 and 3 , respectively.

\section{Numerical method}

The governing equations are changed to a discrete form for an unstructured mesh by the application of Cell Vertex (overlapping) scheme of the Finite Volume Method. This method results in the formulation [7]

$$
W_{i}^{n+1}=W_{i}^{n}-\frac{\Delta t}{\Omega_{i}} \sum_{k=1}^{N_{i}}(\bar{F} \Delta y-\bar{G} \Delta x)_{k}^{n},
$$

where the superscripts $n$ and $n+1$ denote the $n$th and the $(n+1)$ th time stages, $W_{i}^{n}$ represents the conserved variables at the center of control volume $i$ (Figure 1), $\Omega_{i}$ is the area of the control volume, $\bar{F}$ and $\bar{G}$ are the mean values of fluxes on the control volume boundary side $k$, and $N_{i}$ is the number of sides of the control volume. The time step (proportional to the minimum mesh spacing) between time stages $n$ and $n+1$ is denoted by $\Delta t$. In the present study, a three-stage Runge-Kutta scheme stabilizes the computational process by damping high frequency errors, which in turn relaxes the CFL condition [4]. 


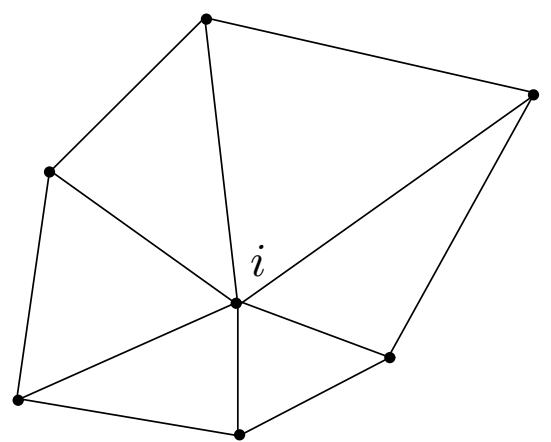

Figure 1: Control volume $i$ is formed by the triangles sharing node $i$.

The explicit solution of above formulation on equally spaced grids presents the behavior of central difference schemes. These schemes do not provide any dissipation mechanism that would compensate the absence of damping by physical viscosity near the high gradient regions. In order to damp unwanted numerical oscillations associated with the explicit solution of the above algebraic equation the numerical dissipation term is added to the convective term $[7]$

$$
C\left(W_{i}\right)=\sum_{k=1}^{N_{i}}(\bar{F} \Delta y-\bar{G} \Delta x)_{k}^{n} .
$$

The numerical dissipation term

$$
D\left(W_{i}\right)=\varepsilon \sum_{k=1}^{N_{i}} \Lambda_{k}\left[L\left(W_{i}\right)-L\left(W_{j}\right)\right]_{k}^{n},
$$

where the Laplacian operator

$$
L\left(W_{i}\right)=\sum_{k=1}^{N_{i}}\left(\nabla W_{i}-\nabla W_{j}\right)_{k} .
$$

The Laplacian operator at node $i$ is computed using $W$ at the end nodes of all edges meeting node $i$. Here $\Lambda_{k}$ is the minimum of $\lambda_{i}$ and $\lambda_{j}$, the scaling 
factors of the edges associated with the end nodes $i$ and $j$ of the edge $k$. This formulation is adopted using the local maximum value of the spectral radius of the Jacobian matrix [7]:

$$
\lambda_{i}=\sum_{k=1}^{N_{i}}\left\{|\bar{u} \Delta y-\bar{v} \Delta x|+\sqrt{|\bar{u} \Delta y-\bar{v} \Delta x|+\beta^{2}\left(\Delta x^{2}+\Delta y^{2}\right)}\right\}_{k} .
$$

Here, $\bar{u}$ and $\bar{v}$ are average velocity component of two nodes of the edge $k$. Here $\varepsilon=1 / 256$ is used to obtain enough numerical dissipation required for stable convergence to the steady state condition with negligible degradation of the accuracy of the solution.

$$
W_{i}^{n+1}=W_{i}^{n}-\frac{\Delta t}{\Omega_{i}}\left[C\left(W_{i}\right)-D\left(W_{i}\right)\right] .
$$

The value $W$ at node $i$ is modified at every time step by subtracting a residual term $R\left(W_{i}\right)=\Delta t\left[C\left(W_{i}\right)-D\left(W_{i}\right)\right] / \Omega_{i}$ which is computed using the quantities $W$ at the nodes of boundary sides of the control volume $i$ (Figure 1). As an edge forms part of the boundary of two control volumes, it is efficient to use the edge-base data structure for definition of unstructured meshes. Using the edge-base computational algorithm reduces the number of times the memory is addressed, and therefore provides a considerable saving in computational time.

\section{Boundary conditions}

The implementation of the flow and solid wall boundary conditions are adopted for the unstructured meshes. For incompressible flow, at the inflow boundaries free stream values of $u$ and $v$ are imposed and $p$ is extrapolated from interior nodes, and at the outflow boundaries free stream pressure is imposed and $u$ and $v$ are extrapolated from interior nodes. The sign of the dot product of the computed velocity vector and normal vector of the flow 
boundary curve at computational nodes is used to distinguish between the in-flow and out-flow boundary.

At solid wall nodes the component of the velocity vector normal to the solid wall boundary edges are set to zero, but since the flow is inviscid, the tangential slipping velocities are not zero and are calculated by a method which suits unstructured meshes.

\section{Domain discretization}

The solution domain is discretized using an unstructured triangular mesh. This method of domain discretization facilitates geometric modeling of the flow fields with complex irregularities in their boundaries. The irregular triangular mesh was produced using Deluaney Triangulation. Such a mesh generation method allows local refinement of triangular elements by using source points and lines. The use of an unstructured mesh for domain discretization enables mesh refinement in regions where high gradients of the flow parameters exists. In addition it provides the ability to use coarser mesh spacing at positions where the gradients of the flow parameters have small magnitudes. This increases the speed of numerical computation [8].

\section{Verification of accuracy}

The accuracy of the incompressible inviscid flow solver is examined by solving two problems with analytic solutions. The first problem is the flow in an internal $90^{\circ}$ corner as shown in Figure 2. The analytical solution is obtained from potential flow theory by using a conformal mapping [10].

The computations were performed on a fully unstructured mesh of triangles (see Figure 2). A unit free stream velocity and pressure was imposed at 
(a)
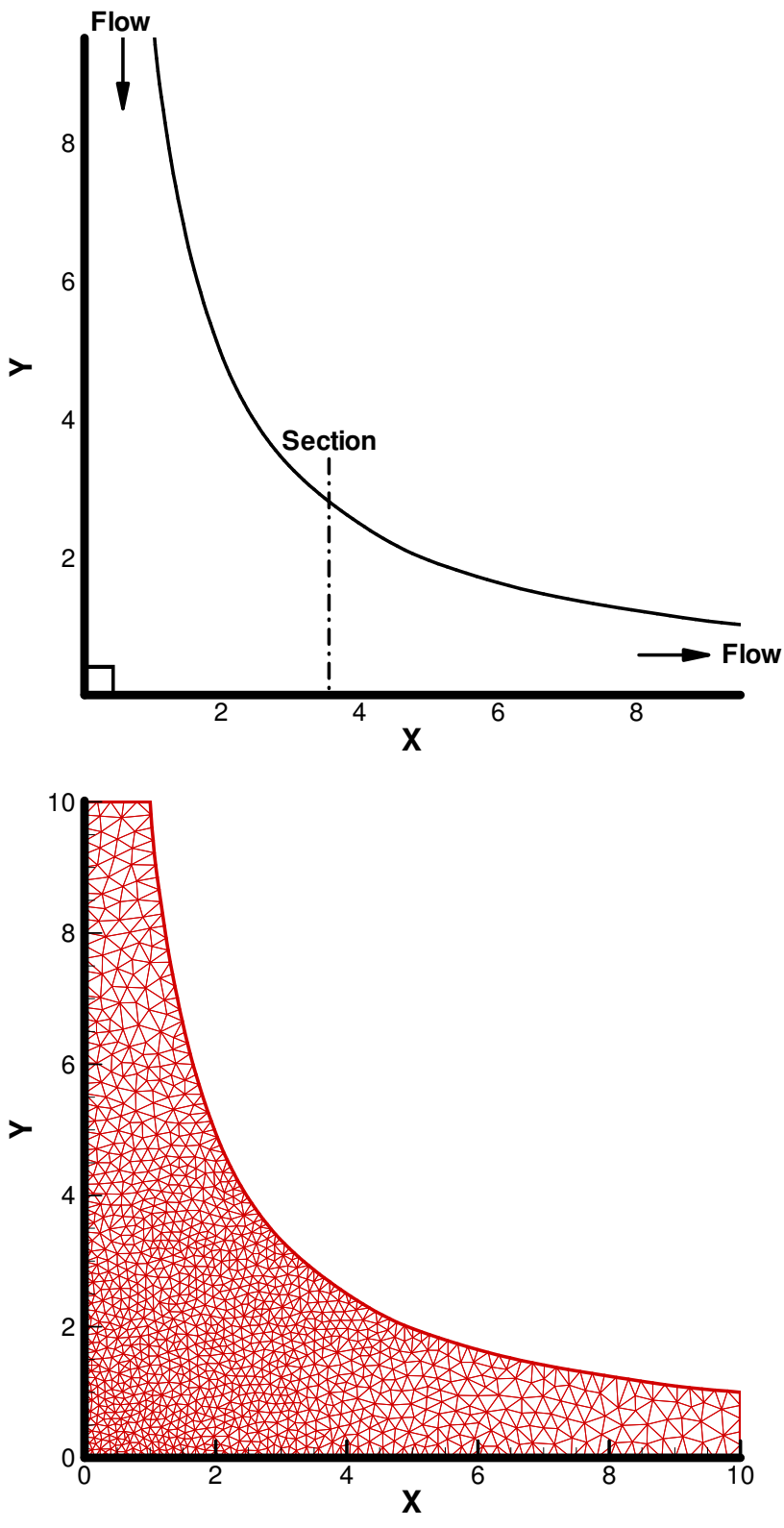

(b)

Figure 2: (a) The flow domain showing the section where the exact and computed solutions are compared; and (b) the unstructured mesh used in the computation. 
(a)

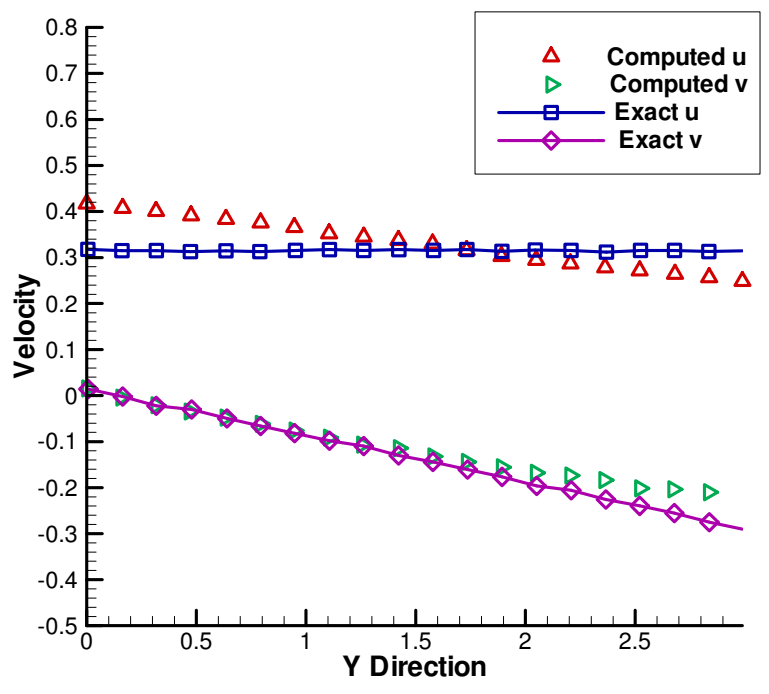

(b)

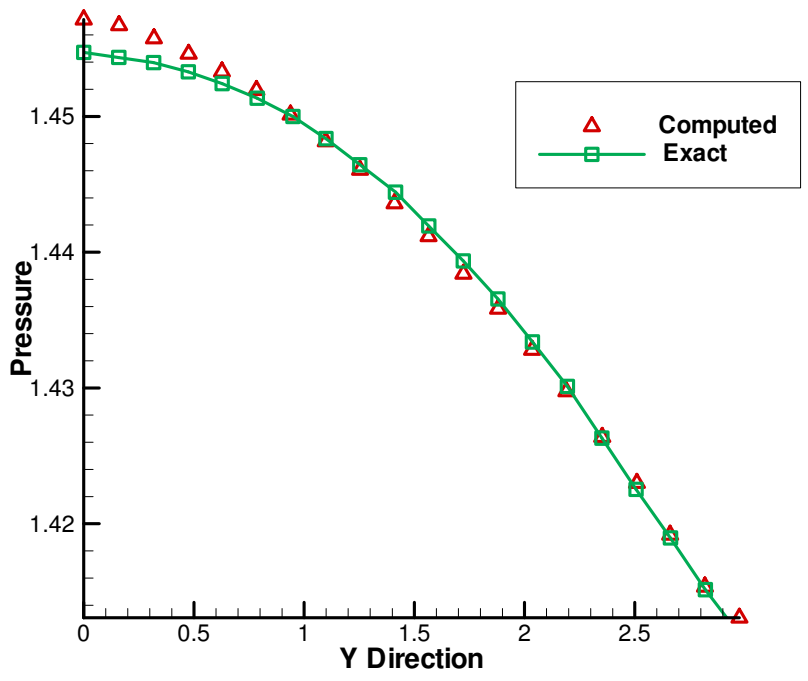

Figure 3: Comparison of the exact and computed values of: (a) velocity components; and (b) pressure on the section shown in Figure 2(a). 


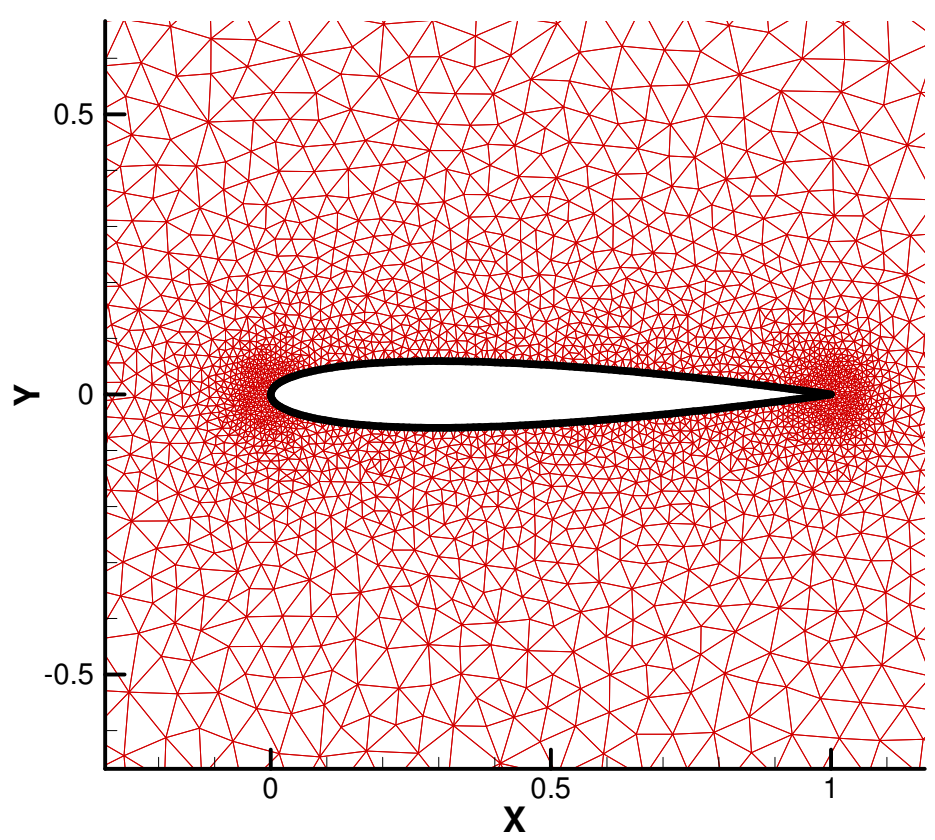

Figure 4: Part of the discrete flow domain for the calculation of the flow around a NACA0012 aerofoil.

inflow and outflow boundaries respectively. A comparison of computed velocity components and pressures with the exact solution in a section shown in Figure 2 may be found in Figures 3. Despite the irregular distribution of grid spacing in the unstructured mesh, the symmetric turning flow is accurately computed and a stagnation point is correctly formed at the corner. The computed results demonstrate the accuracy of the algorithm to compute a flow fields experiencing both stagnant and turning conditions.

The second problem is to calculate the incompressible inviscid flow around a NACA0012 aerofoil for which both experimental measurements and exact solutions are available $[6,5]$. The mesh may be seen in Figure 4. 


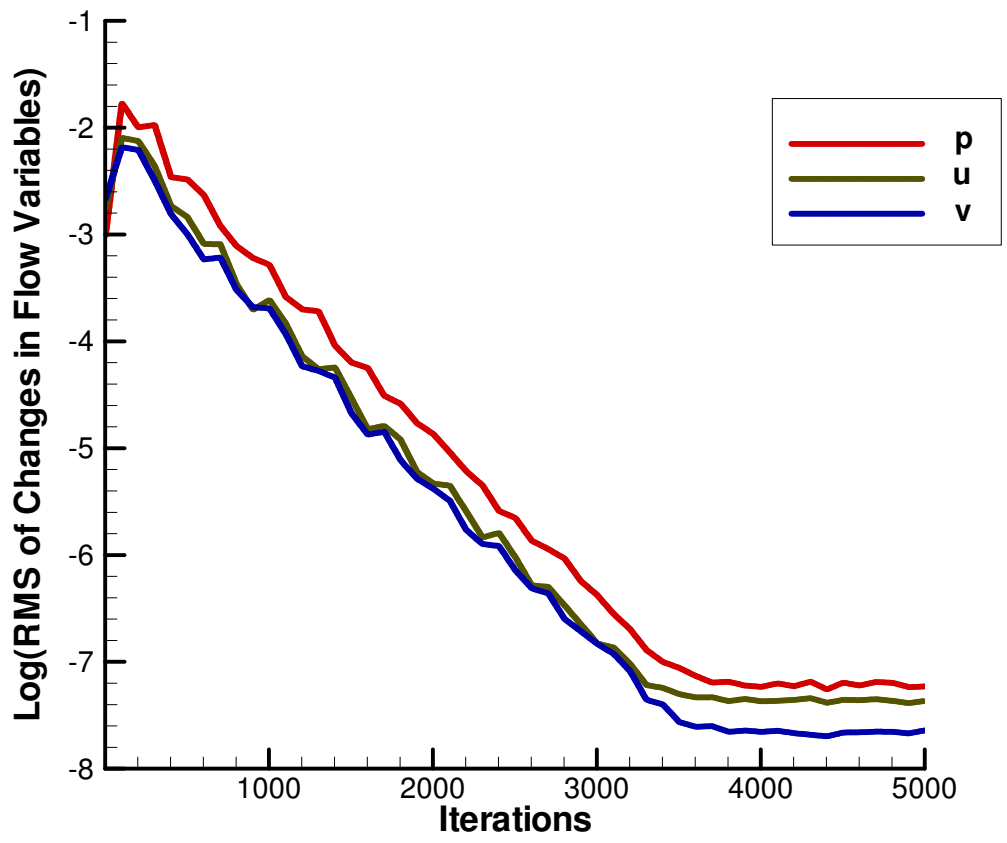

Figure 5: Convergence behavior. 
The flow is calculated for angle of incidence of $5^{\circ}$. A unit free stream velocity and pressure is imposed at the inflow and outflow boundaries of the computational mesh. The free stream flow parameters (outflow pressure and inflow velocity) are set at every computational node as initial conditions. The typical convergence of the flow parameters toward the steady state condition is demonstrated in terms of root mean squares of change in pressure and velocity components (Figure 5). This shows the good convergence properties of the scheme.

The results for the case of free stream velocity with $5^{\circ}$ incidence may be seen in Figure 6(a). The comparison of the computed results with exact values and experimental results in terms of the coefficient of pressure $C_{p}=$ $\left(p-p_{0}\right) /\left(0.5 \rho_{0} U_{0}^{2}\right)$ on the aerofoil boundaries demonstrates the accuracy of the model (Figure 6(b)). The pressure contours are not symmetric and the agreement between the computed and both the exact solution [5] and experimental measurements [6] is encouraging.

\section{Discussion}

An algorithm that uses the cell vertex finite volume method with an unstructured triangular mesh to determine incompressible inviscid flows has been developed and shown to produce results that are in excellent agreement with exact results for two benchmark problems. The pseudo compressibility method is used to overcome the numerical problem associated with the solution of the equations of continuity and motion. Adding a pressure time derivative term to the continuity equation successfully couples the pressure and velocity fields and speeds up the convergence behavior of the explicit solution procedure without any degradation in the accuracy of the results. Such an efficient algorithm for the computation of both velocity and pressure fields facilitates the numerical modelling of the incompressible inviscid flow problems on complex domains. 
(a)

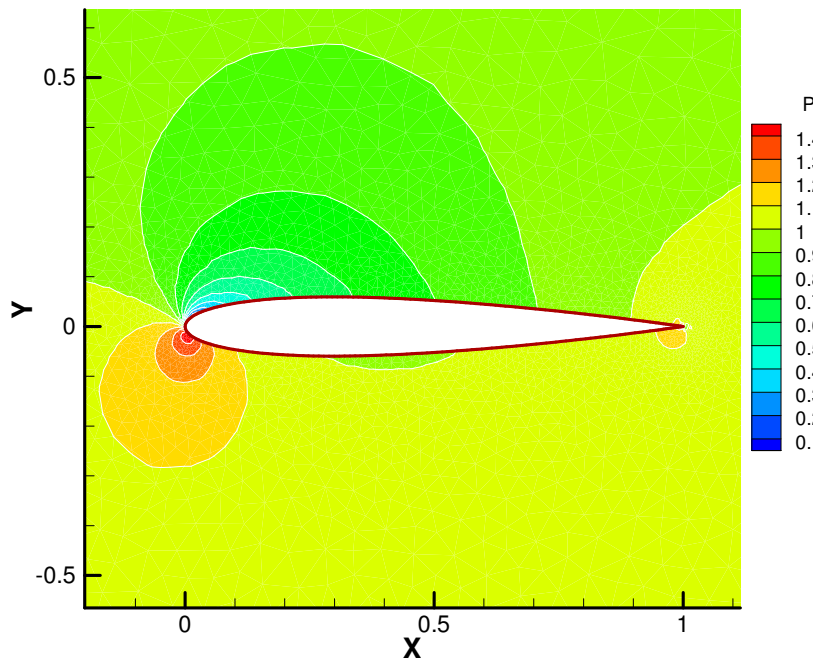

(b)

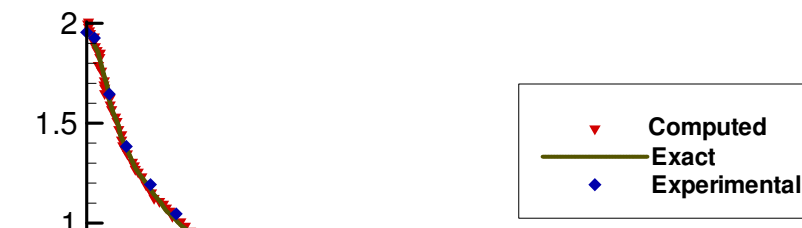

FigURE 6: Results for NACA0012 aerofoil at $5^{\circ}$ incidence: (a) computed pressure contours; and (b) comparison of computed, experimental and exact values of $C_{p}$. 


\section{References}

[1] J. L. Chang and D. Kwak, On the method of pseudo compressibility for numerically solving incompressible flow, AIAA 84-0252, 22nd Aerospace Science Meeting and Exhibition, 1984, Reno. C1226

[2] A. Chorin, A numerical method for solving incompressible viscous flow problems, Journal of Computational Physics, Vol. 2, 1967, 12-26. C1224, C1225, C1226

[3] J. Dreyer, Finite volume solution to the steady incompressible euler equation on unstructured triangular meshes, MSc Thesis, MAE Dept., Princeton University, 1990. C1227

[4] A. Jameson, W. Schmidt and E. Turkel, Numerical solution of the euler equations by finite volume method using Runge-Kutta time stepping schemes, AIAA Paper 81-1259, AIAA 14th Fluid and Plasma Dynamics Conference, California, 1981. C1227

[5] J. Peraire, Potential flow past arbitrary aerofoils using conformal mapping, Private Communication. C1233, C1235

[6] L. Eriksson, Calculation of two dimensional flow wall interface for multi-component airofoil in closed low speed tunnel, The Aeronautical Research Institute of Sweden FFATN AU-1116, Part 1, Stockholm, 1975, C1233, C1235

[7] S. R. Sabbagh-Yazdi and A. Hadian, Pressure-velocity coupled finite volume solution of steady incompressible flow using artificial compressibility technique, International Journal of Engineering, Transaction A: Basics, Vol. 17, No. 2, June 2004, 109-118. C1227, C1228, C1229

[8] J. F. Thompson, K. Soni-Bharat and N. P. Weatherill, Handbook of grid generation, CRC Press, New York, 1999. C1230 
[9] E. Turkel, Preconditioning methods for solving the incompressible and low speed compressible equations, ICASE Report, 1986, 86-14 C1227

[10] H. R. Vallentine, Applied Hydrodynamics, Butterworths, S.I. Edition, London, 1969. C1230 